\title{
Diagnostic and Prognostic value of Immunohistochemistry in Destructive Paediatric Maxillary Pathologies
}

Priya Jeyarai

Commanding Officer, Military Dental Centre (Gough Lines), Secunderabad, Telangana, Pin- 500015, India.

Article Info

\section{Article Notes}

Received: August 19, 2020

Accepted: October 06, 2020

\section{${ }^{*}$ Correspondence:}

Dr. Priya Jeyaraj, Commanding Officer, Military Dental Centre (Gough Lines), Secunderabad, Telangana, Pin- 500015, India; Email: jeyarajpriya@yahoo.com

C 2020 Jeyaraj $P$. This article is distributed under the terms of the Creative Commons Attribution 4.0 International License.

\section{Keywords:}

Immunohistochemistry $(\mathrm{IHC} / \mathrm{H})$

Histopathological Examination (HPE)

Unicystic lesions

Dentigerous Cysts

Unicystic Ameloblastoma

Odontogenic Keratocyst (OKC)

Calretinin; Ki-67

Proliferating Cell Nuclear Antigen (PCNA)

Proliferation Index $(\mathrm{PI})$

Labelling Index (LI)

\section{ABSTRACT}

Introduction: Establishing an accurate diagnosis and probable prognosis of ambiguous, extensive and destructive maxillary pathologies, is imperative for an appropriate, timely and effective treatment modality to be instituted. This is particularly true in the paediatric population, in order to ensure complete elimination of the lesion, with the least possible morbidity, debility, or interference with normal jaw growth.

Objectives: To assess the diagnostic and prognostic value of Immunohistochemistry (IHC) as an adjunct to Histopathological examination (HPE), in cases of destructive paediatric maxillary pathologies. To use the information thus obtained, to select the most ideal and efficacious management protocol for each case.

Material \& Methods: An extensive study was carried out on 25 cases of destructive (as evidenced clinically and radiographically) maxillary lesions, in children of ages between 5 and 16 years. Positivity for an IHC tumor marker, namely Calretinin, was employed to distinguish maxillary cysts from tumors. In addition, Labelling indices of two IHC cell proliferation markers, namely Ki-67 and PCNA, indicated the proliferative activity of constituent cells of the pathologies, which aided in predicting their aggressive nature and recurrence potential. On the basis of the above information, the choice between a conservative versus radical treatment approach was made for each individual case.

Results \& Conclusion: IHC proved to be of immense value as a diagnostic marker and a prognostic indicator in the paediatric maxillary pathologies. In addition to aiding the pathologist in making an accurate confirmatory diagnosis, it also served as an invaluable tool to the surgeon, in guiding the treatment plan by indicating the likely prognosis and chances of recurrence of these lesions.

\section{Introduction}

There often exists ambiguity in the diagnosis and management of large, destructive pathologies involving the maxilla in children ${ }^{1,}$ ${ }^{2}$. Most of these destructive maxillary pathologies in the paediatric population, are either inflammatory, odontogenic or developmental in origin, arising from either the tooth forming apparatus or from epithelial remnants trapped within closing sutures of the craniomaxillofacial complex. They range from cysts and hamartomas to benign and even malignant neoplasms. Overlapping clinical, radiographic and histopathological features between the various lytic lesions such as Odontogenic cysts and tumours, compound the challenges and difficulties faced in distinguishing them from one another. Different pathologies vary widely in their clinical behaviour, aggressive and invasive potential, and tendency for recurrence and malignant transformation ${ }^{3,4,5}$, hence requiring vastly different management protocols. 
HPE has remained to this day, the Gold Standard for making a confirmatory diagnosis of oral lesions, including destructive maxillary pathologies. On occasions, deceptively innocuous appearing unilocular maxillary lesions have unexpectedly been found to be ominous pathologies such as, benign or even malignant tumors ${ }^{2}$. Relative similarities in their clinical and radiographic presentation, as well as in their histological appearance, makes it both challenging as well as important to distinguish between the two, for an appropriate and timely management to be initiated ${ }^{3}$. It is at such times that IHC steps in as a valuable adjunct to HPE, aiding in an accurate identification of the pathology for an ideal treatment planning, thereon.

Too radical a treatment approach for an indolent and benign pathology can result in undesirable and unnecessary esthetic and functional morbidity in the child, while under-treatment of an aggressive pathology can lead to its persistence, recurrence or even malignant transformation. A precise and correct diagnosis is thus imperative for the institution of an ideal management protocol, in order to completely and effectively eliminate the pathology, while at the same time, ensuring the least possible morbidity, debility and risk of recurrence ${ }^{3}$.

IHC is the science of detection and localization of specific antigens (such as endocrine hormones, oncogene products or cell cycle participants) in biological tissue samples, by using labeled antibodies as reagents ${ }^{4}$. The ensuing AntigenAntibody (Ag-Ab) interactions, resulting in the formation of immunological complexes, can be visualized using markers such as fluorescent dyes, chromogens, enzymes or colloidal gold.

IHC can be employed in two possible ways in the diagnosis and treatment planning of ambiguous maxillary pathologies in children ${ }^{4}$. One is, in their confirmatory identification by Tumor Immunodiagnosis, using 'Tumor Markers' (such as Calretinin, which is specific for odontogenic tumors like Ameloblastoma) that help to distinguish maxillary odontogenic cysts from odontogenic tumors ${ }^{5}$. The other is, evaluation of the proliferative activity of constituent cells of the pathology, by assessing their expression of various 'Cell Proliferation Markers' (Cell cycle associated antigens such as Ki-67 and PCNA) ${ }^{6}$, that give valuable information on the likely prognosis of the maxillary lesions.

Calretinin, is an intracellular antiapoptotic protein and a 'Tumor Marker', which is consistently expressed in all variants of Ameloblastoma and some cases of Keratocystic odontogenic tumors (KCOTs), whereas in none of the other cysts or tumors $5,7,8,9$, thus aiding in their differential diagnosis. In this Study of 25 cases of destructive maxillary pathologies in children, expression of Calretinin helped in distinguishing cystic lesions from odontogenic tumors, and in establishing a confirmatory diagnosis of ambiguous pathologies.
The biological behavior and probable prognosis of destructive maxillary pathologies in children, is rather difficult to predict on the basis of their clinical, radiographic and histological assessment alone $\mathrm{e}^{10,}{ }^{11}$. Immunohistochemical expression of 'Cell Proliferative Markers / Cell cycle associated antigens' like Ki-67, PCNA (Proliferating Cell Nuclear Antigen), AgNORs (Silver staining Nucleolar Organizer Regions) ${ }^{4}$, can give valuable information in this aspect, as presumably, aggressiveness of any lesion is directly proportional to the proliferative activity of its constituent cells'. In this Study, 'Ki-67' and 'PCNA' were employed to assess the aggressive potential of each of the paediatric maxillary pathologies.

An extensive study has been carried out in 25 cases of large, destructive unilocular radiolucent lesions involving the maxilla in a paediatric population (age range of 5 to 16 years), over a period of two years. Details of the Immunodiagnostic procedures employed, management protocols implemented, results achieved and follow up findings have been presented in this study.

\section{Aim And Objectives}

Aim: To establish the value of IHC as a Diagnostic Marker to clinch the diagnosis and as a Prognostic Indicator to predict the aggressive potential of 25 cases of extensive maxillary lesions in children in the age range of 5 to 16 years, and thereby to formulate and institute the most ideal and appropriate treatment modality for each case.

\section{Objectives}

a) To test the correlation between the histopathological typing of the paediatric destructive, unilocular maxillary lesions with their IHC analysis by using a 'Tumor Marker', namely Calretinin, in order to distinguish cysts from the tumors, and to arrive at a precise and conclusive confirmatory diagnosis.

b) To select the most appropriate and effective treatment option for each case, based on the information on probable prognosis provided by the IHC expression of two 'Cell Proliferation Markers', namely Ki-67 and PCNA, by the constituent cells of the pediatric maxillary pathologies.

\section{Materials And Methods}

Sample and Sample Size: Twenty-five cases of large, unilocular, destructive, unilocular, radiolucent lesions of the maxilla, in a paediatric population in the age range of 5 to 16 years, were managed over a period of two years.

Ethical Clearance and Informed Consent: All procedures performed on the patients (human participants) involved in this Study, were in accordance with the ethical standards of the institution and national research committee, as well as with the 1964 Helsinki 
declaration and its later amendments and comparable ethical standards. Institutional Ethical committee clearance was obtained prior to commencing the Study. This Study does not contain any new studies with human participants or animals performed by the author.

Informed written consent was obtained from all the individual participants in this study, including parents of the minors.

In all the 25 cases of destructive maxillary pathologies, HPE and IHC analysis were carried out of the biopsy specimens, in order to establish a confirmatory diagnosis and also to assess the clinical nature of the lesions, in order to formulate an ideal treatment plan for each individual case. Calretinin, an IHC Tumor marker, was employed to distinguish between Odontogenic cysts and tumours. Ki-67 and PCNA, both IHC Cell Proliferation markers, provided information on the biologic behaviour and aggressive potential of these lesions.
On the basis of the above information, an accurate diagnosis was arrived at and the most appropriate definitive management protocol was established for each of these different lesions on a case to case basis, in order to achieve the most ideal functional and esthetic outcome (Figures 1-12).

HPE and IHC Analysis: Average size of the tissue samples tested were $1 \mathrm{~cm}$ X $0.5 \times 0.5 \mathrm{~cm}$. All samples were formalinfixed and paraffin embedded. The blocks were sectioned using a cryotome, into 4 to $7 \mu \mathrm{m}$ thick sections, that were placed on Super Frost slides for the Haematoxylin-Eosin and IHC staining. HPE was performed using HaematoxylinEosin stained sections observed under magnifications of 10X, 50X and 100X. IHC studies were carried out, by staining for Calretinin, Ki-67 and Proliferating Cell Nuclear antigen (PCNA).

IHC Technique: Labelled secondary antibodies were used to detect the three antigens of interest. The Avidin-
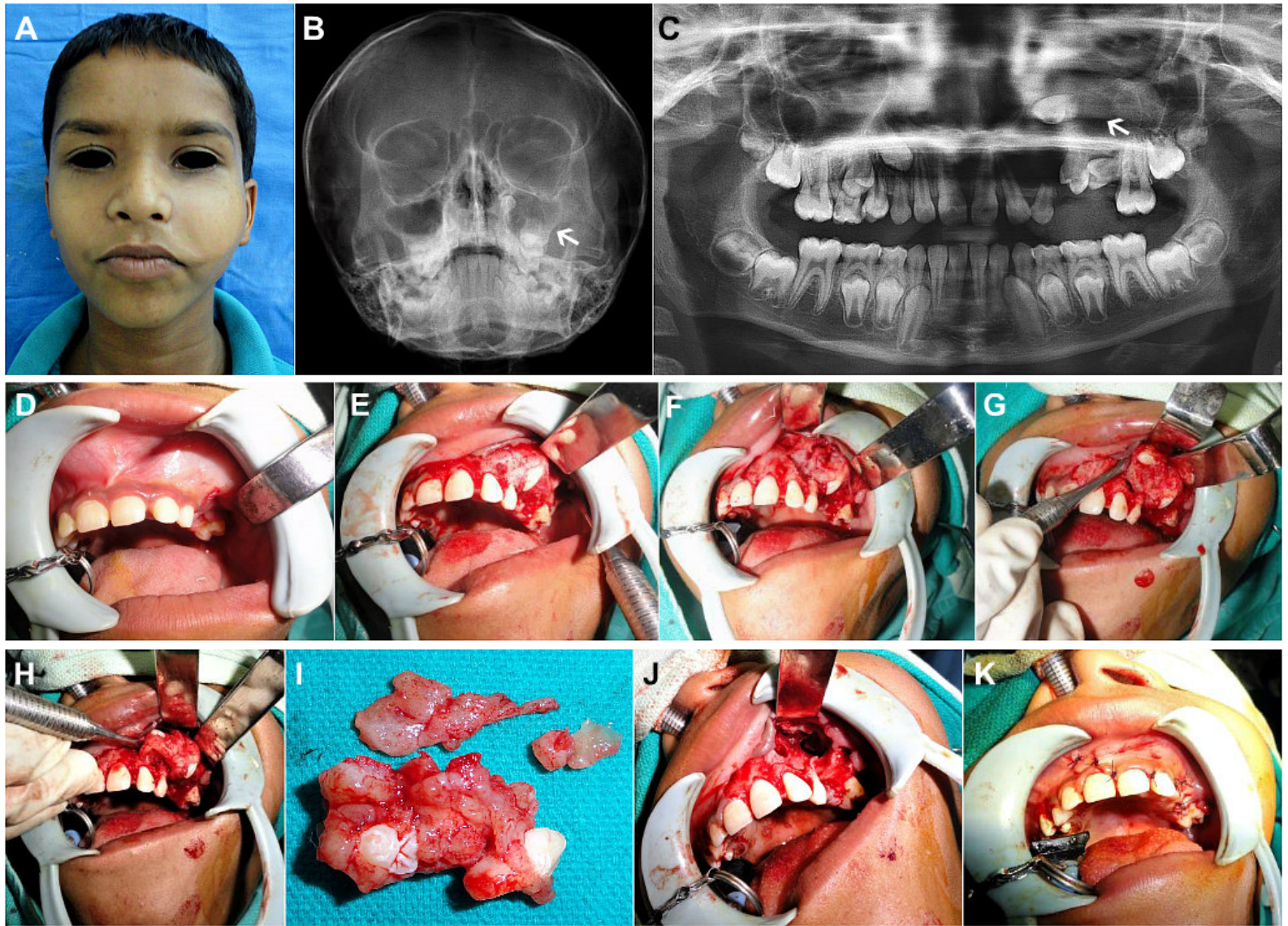

Figure 1: (Case 1) (A) 9yr old child with a painless, slow growing, expansile, firm swelling over the left cheek. (B, C) Radiographs Occipitomental view of skull and Orthopantomogram (OPG), revealed a large unilocular radiolucency involving the left maxilla and antrum (as indicated by the white arrows), associated with 2 unerupted premolar teeth. (D-F) Following histological confirmation of Dentigerous cyst by incisional biopsy, the patient was taken up for enucleation and curettage after deroofing of the cystic lesion via an intraoral upper buccal vestibular approach. $(G, H)$ The lesion shelled out easily from the underlying bony cavity and was removed in toto along with the two teeth within its lumen. (I) Gross appearance of the excised cystic lesion with the unerupted tooth germs teeth within. $(\mathrm{J}, \mathrm{K})$ Antral lining completely extirpated, peripheral ostectomy of the antral walls carried out using vulcanite trimmers, preserving the integrity of the infraorbital nerve, chemical cauterisation of antral walls, followed by closure of the mucoperiosteal flap. 


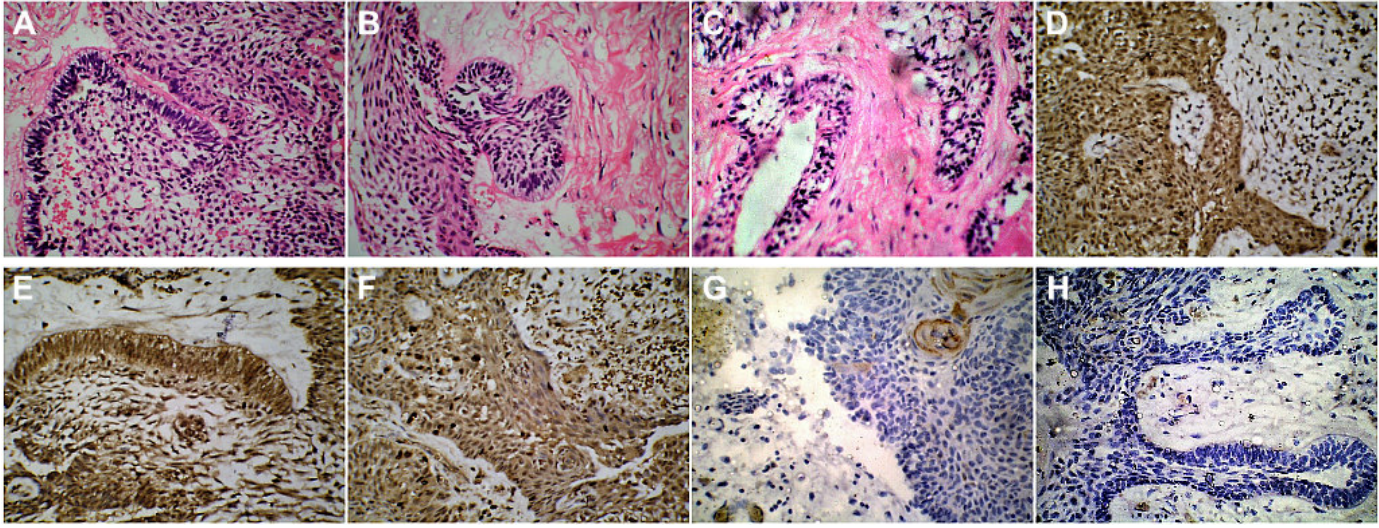

Figure 2: (Case 1) HPE and IHC demonstrating Ameloblastomatous transformation of a Dentigerous Cyst. (A-C) H\&E stained sections (magnification X50) of the enucleated cystic maxillary lesion, showing a hyperplastic epithelial lining and a fibrous capsule with some chronic inflammatory cell infiltrate. Few areas of the epithelial lining showed the typical tall columnar basal cells with reverse palisading of the nuclei, indicative of an ameloblastomatous change. These ameloblastomatous areas also showed invagination into the lamina propria in some locations. (D-F) Immunohistochemical examination (magnification X50) of the biopsy specimen revealed Calretinin positivity expressed by the epithelial cells, suggestive of their ameloblastomatous transformation. (G) A positive, but low Ki-67 Labelling Index of 1.7 expressed by the cells in the epithelial zone. (H) A low PCNA Proliferative Index of 1.3, indicative of a lesion with low invasive potential.
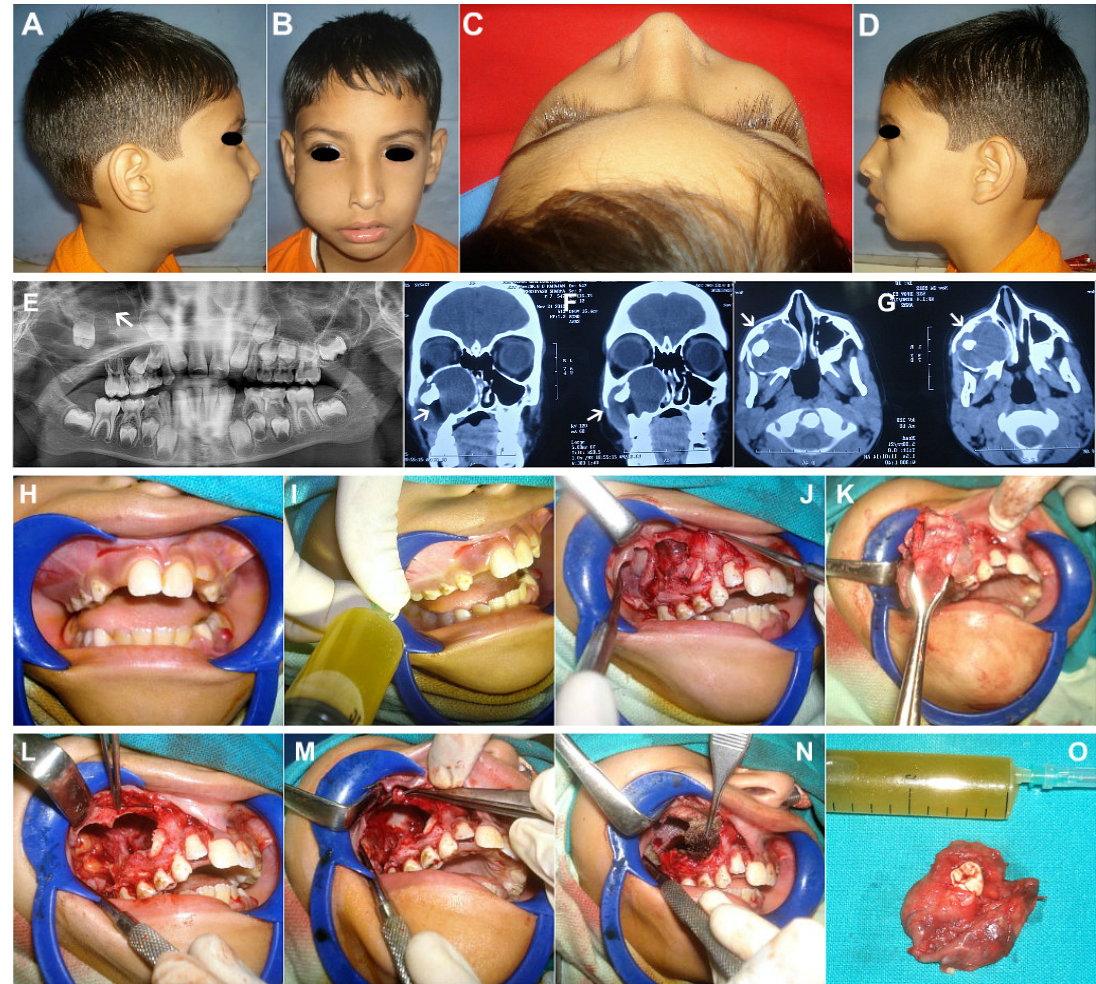

Figure 3: (Case 2) (A-D) 8yr old patient with a slowly expanding bony swelling in the right infraorbital and cheek region, causing obliteration of the nasolabial fold. (E-G) OPG and Coronal \& Axial sections of Non Contrast Computed Tomographic Scans (NCCT), showing a large unilocular expansile lesion, filling up the entire right maxillary antrum and extending laterally by perforation of the anterolateral wall, and medially into the nasal cavity. Orbital floor (roof of the antrum) appears intact. A displaced second molar tooth germ was seen within the large lytic lesion, giving the impression of a Dentigerous cyst. (H) Expansion of the buccal cortical plate evident in left molar region. (I) Aspiration of clear straw coloured cystic fluid from within the cystic lesion. (J) Mucoperiosteal flap raised exposing the thinned out cortical bone with perforations, revealing the cystic lining of the lesion within. (K) Cyst enucleated in toto along with its entire lining. (L) The entire antral lining was carefully extirpated as well, as the lesion was an IH proven case of Unicystic Ameloblastoma. (M) Infraorbital Nerve carefully dissected and preserved. (N) Chemical cauterization of the bony defect and walls of the maxillary antrum carried out using Carnoy's solution, after carefully isolating the Infraorbital Nerve and the adjacent soft tissues using Vaseline gauze. (O) Gross appearance of the enucleated pathological specimen with the unerupted second molar tooth germ within, and the clear straw coloured cystic fluid aspirate. 

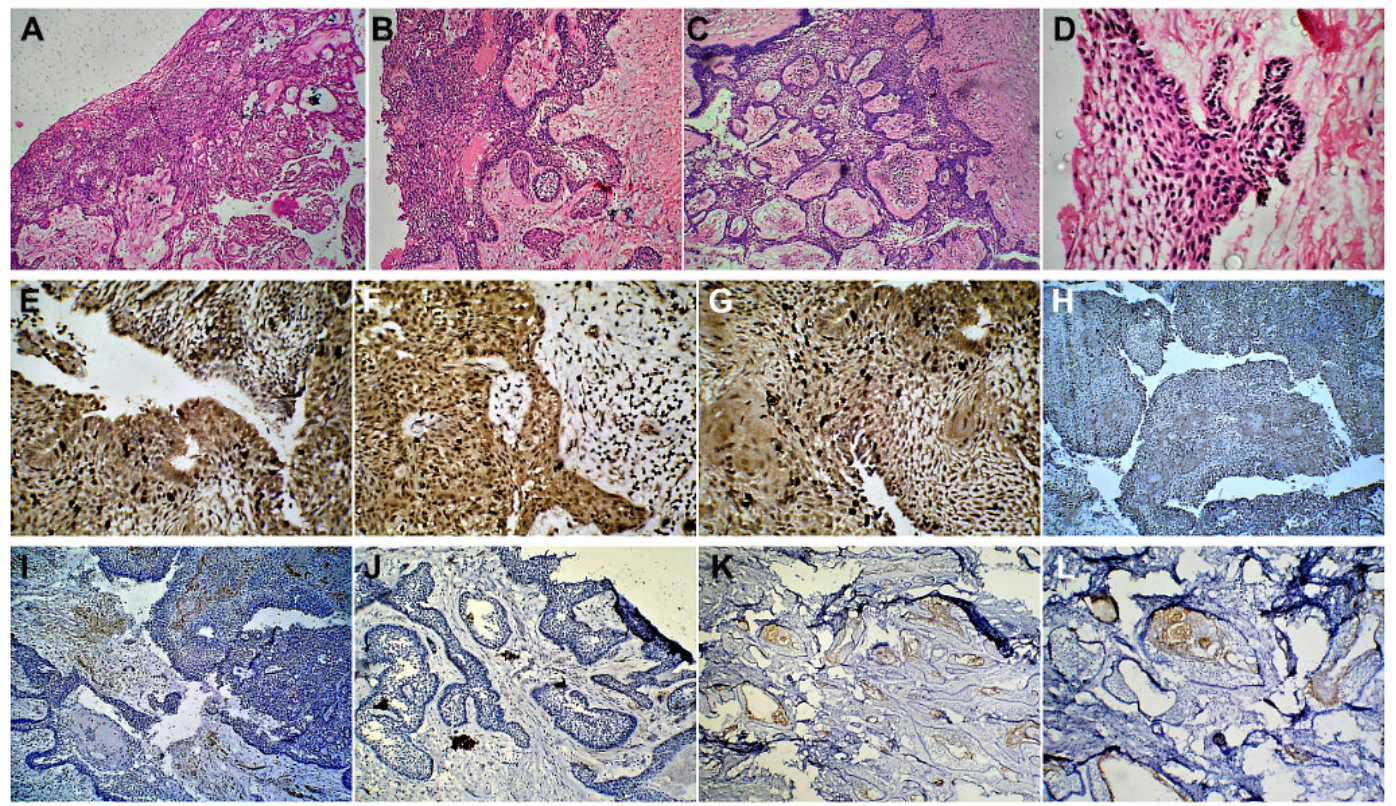

Figure 4: (Case 2) (A-D) Histopathological examination of H\&E stained sections (magnification X50), revealed the cystic cavity surrounded by a dense fibrous capsule. The cavity was lined by a hyperplastic epithelial lining comprising of a basal cell layer composed of typical tall columnar ameloblastic cells with reverse palisading of their nuclei, indicative of a Unicystic Ameloblastoma. (E-G) Immunohistochemical examination of the biopsy specimen (magnification X50) revealed Calretinin positivity expressed by the epithelial cells, confirming the diagnosis of Unicystic Ameloblastoma. Positivity was expressed by both, the peripheral tall columnar ameloblast-like cells as well as the central areas of stellate reticulum like cells. $(\mathrm{H}, \mathrm{I})$ A positive, but low Ki-67 Labelling Index of 2.1 expressed by the cells in the epithelial zone. (J-L) A low PCNA Proliferative Index of 1.8, indicative of a lesion with a low invasive potential.
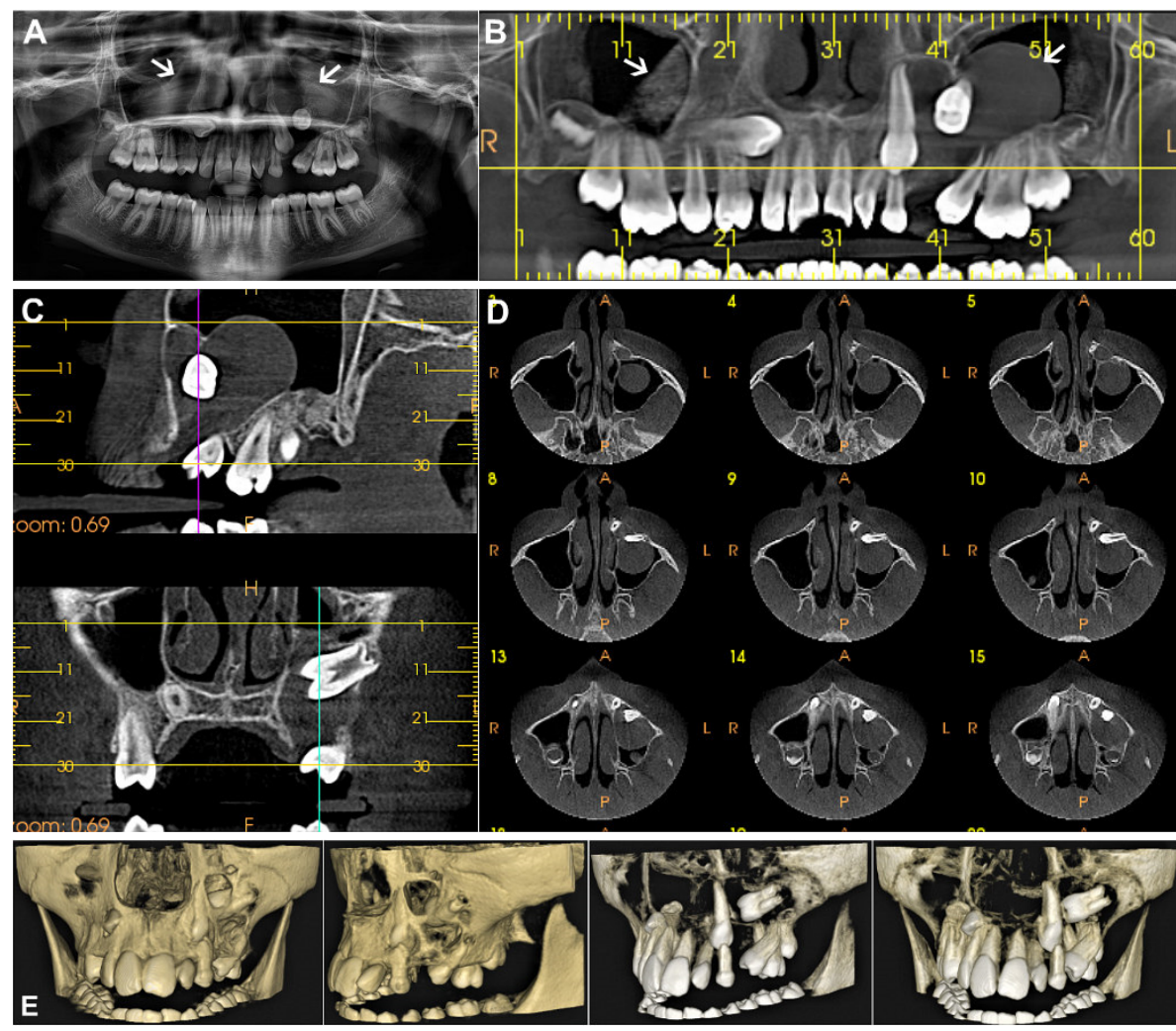

Figure 5: (Case 3) (A) OPG and (B-D) Cone beam computed tomographic (CBCT) scans of the maxillofacial region in a 10yr old female patient, showing bilateral unilocular cystic maxillary lesions occupying the maxillary antra on both sides. The cystic pathologies were found to be engulfing multiple unerupted and displaced tooth germs. (E) 3-D Reformatted images showed destruction of bone and perforation of the buccal cortical plate by the maxillary pathology, and the presence of multiple displaced teeth germs within it. 

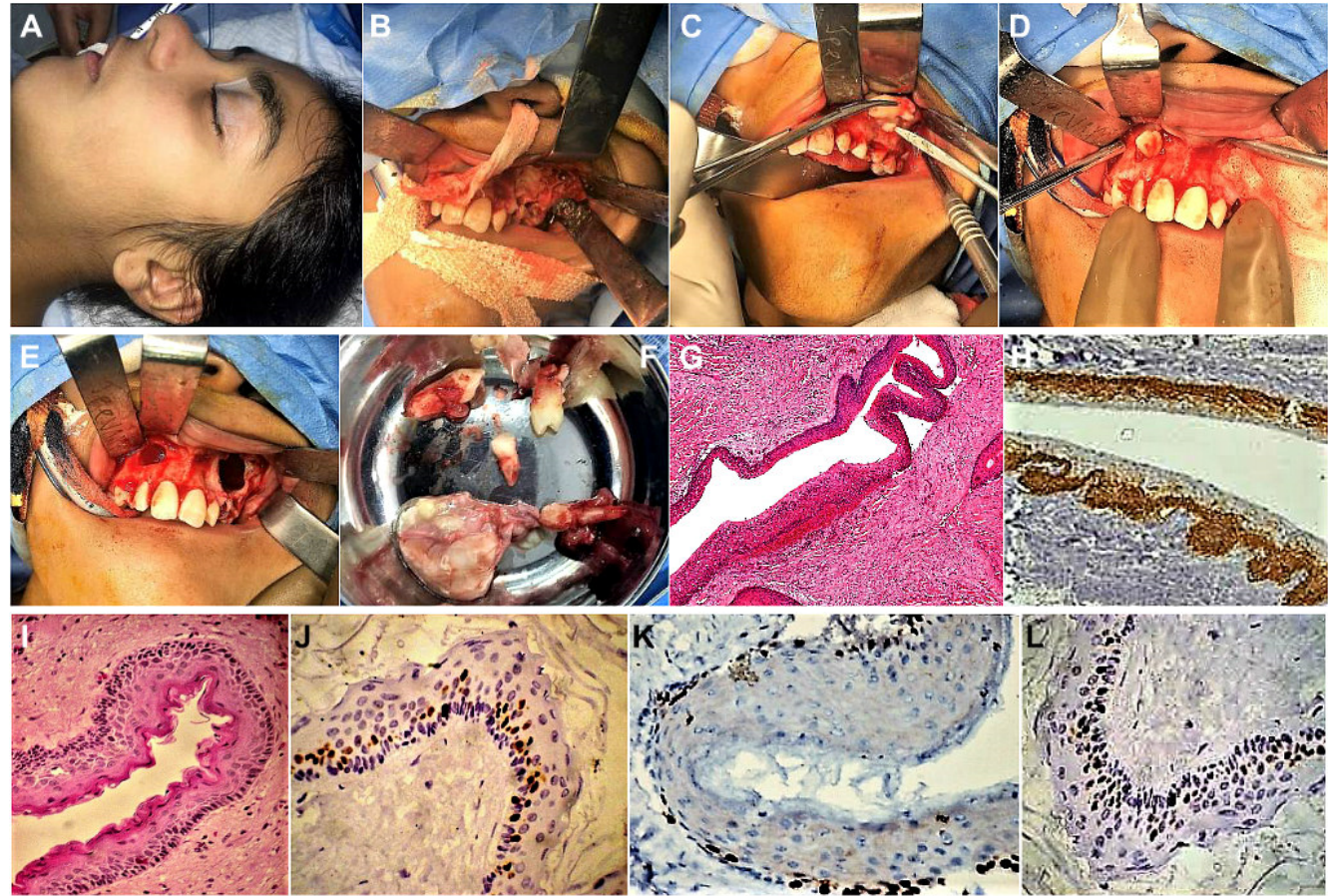

Figure 6: (Case 3) (A) Patient taken up for surgery under General Anaesthesia (GA). (B-E) Upper labial mucoperiosteal flaps raised bilaterally, exposing the perforated buccal cortical plates. Deroofing and exposure of the bilateral maxillary cystic lesions accomplished by removing windows of cortical bone from both sides of the maxilla. Lesions enucleated along with the associated tooth germs within, followed by curettage and chemical cauterisation of the antral walls with Carnoy's solution, followed by closure of the mucoperiosteal flaps. (F) Gross specimens of the excised lesions. (G) H \& E stained sections (magnification X50) showing the cyst wall lined by corrugated layer of parakeratinized stratified squamous, suggestive of a Keratocystic Odontogenic Tumor (KCOT). (H) Positive IH staining for Calretinin (magnification X50), confirmatory of KCOT. (I) H \& E stained sections (magnification X 500) showing the cyst wall lined by corrugated layer of parakeratinized stratified squamous epithelium with a distinct granular cell layer. Flakes of keratinised material were noted within the cyst lumen, (J) IHC analysis (magnification X1000) showing occasional staining of a few basal cells for Ki-67 (Low LI values of 0.5) (K, L) IHC positivity expressed by few basal cells of the epithelial lining for PCNA (Low LI values of 0.7 ), indicative of the presence of a proliferative potential, albeit low.
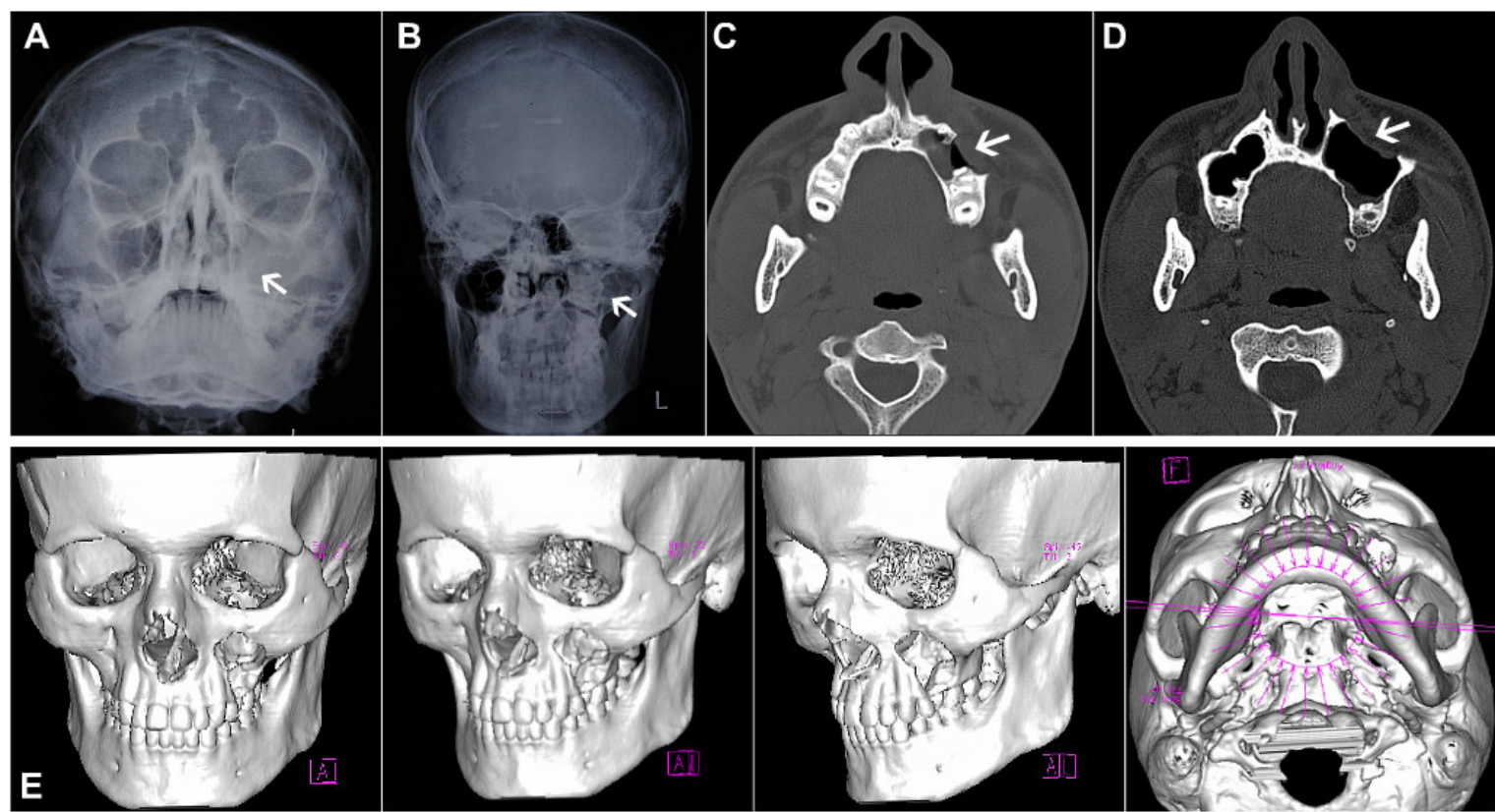

Figure 7: (Case 4) (A-E) Radiographs and NCCT of an extensive unilocular lesion of the left maxilla, with antral involvement, in a 14yr old male patient. Destruction of the overlying buccal cortical plate is evident on sagittal and 3-D reformatted CT images. 

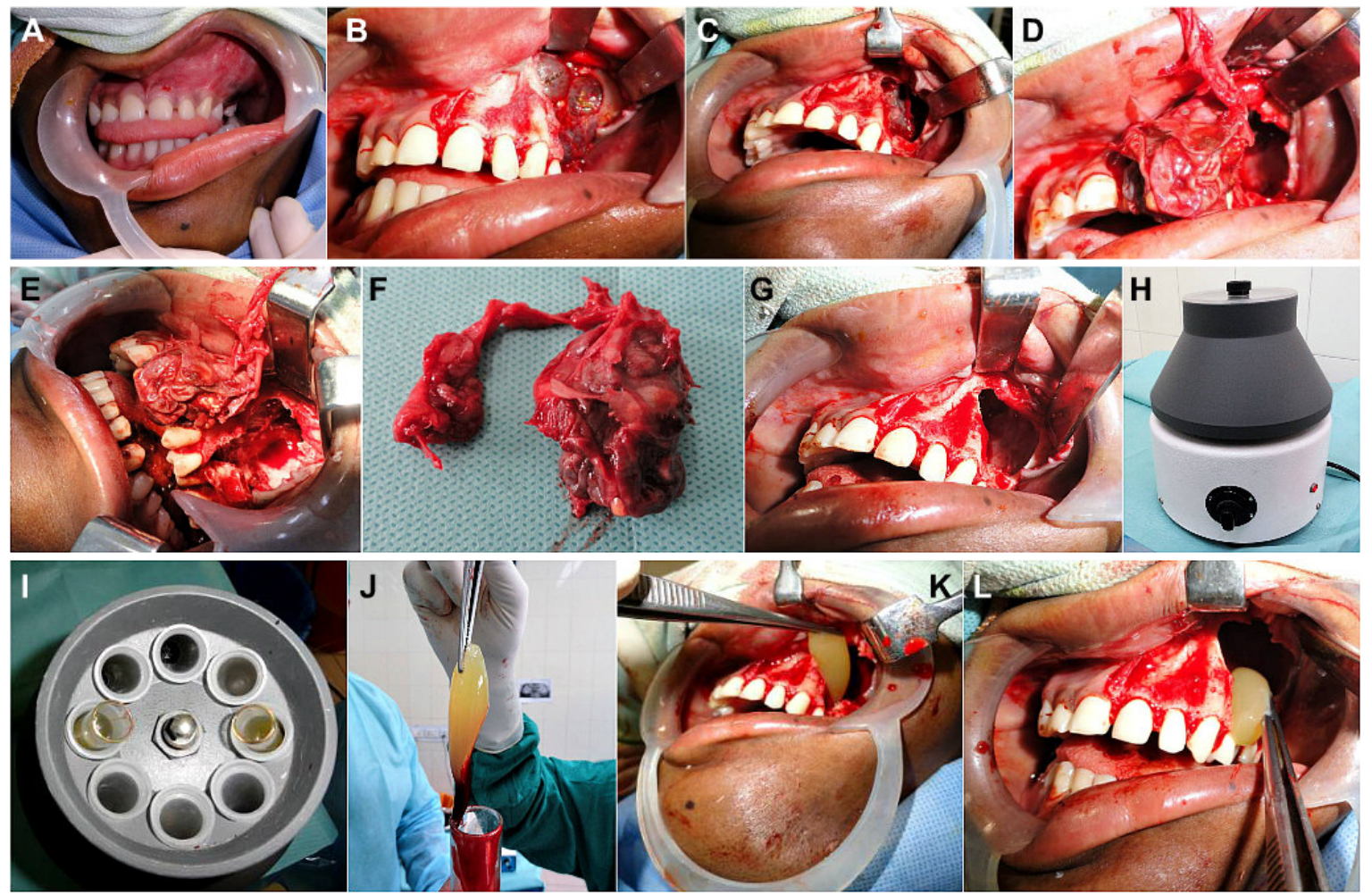

Figure 8: (Case 4) (A) A 14yr old patient with a large maxillary lesion producing expansion of the upper left buccal cortical plate in the premolar-molar region and obliteration of the buccal vestibule in the region. Incisional biopsy carried out earlier, had confirmed the diagnosis of a Dentigerous cyst, so the patient was planned for enucleation and curettage under GA. (B) On raising a full thickness mucoperiosteal flap, the expanded buccal cortical plate, as well as areas of its thinning and perforations caused by the expanding pathology within, were visualized. Bluish translucent areas of the underlying cyst, were also visible. (C) Complete deroofing of the cystic lesion was carried out by removing the overlying spurs of buccal cortical plate, and nibbling the bony edges of the cavity, using bone rongeurs. (D, E) Enucleation of the lesion was carried out, taking care not to leave any portion of the cystic lining behind. This was followed by thorough curettage of the cavity walls. (F) Gross specimen of the enucleated cyst. (G) The antral lining of the left maxillary sinus was completely extirpated followed by thorough antral lavage. $(\mathrm{H}-\mathrm{L}) \mathrm{A}$ centrifuge was used to prepare fresh autologous Platelet Rich Fibrin (PRF), which was then placed into the bony cavity to enhance soft tissue healing and hasten subsequent bone fill and reossification of the lytic area.
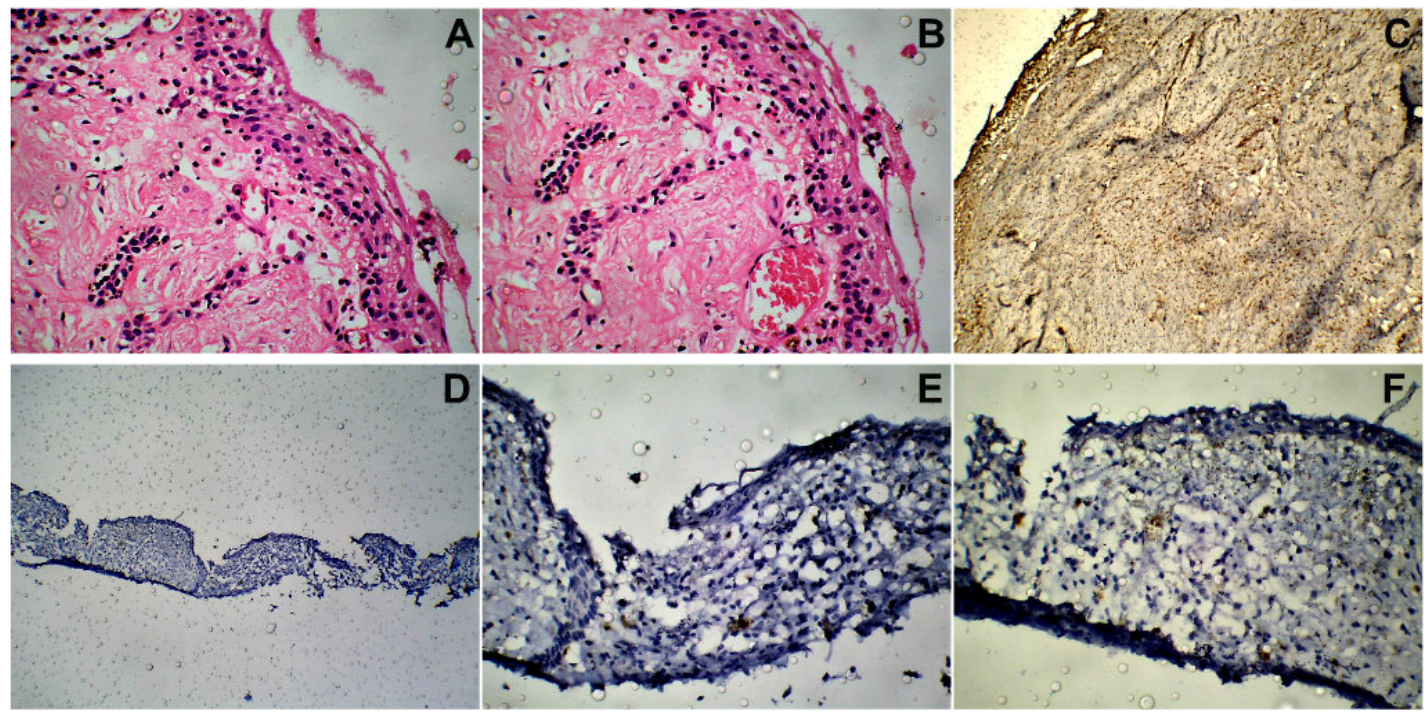

Figure 9: (Case 4) (A, B) H \& E sections (magnification X100). Histopathological examination showed features of a Dentigerous cyst, such as the cyst lining composed of stratified squamous epithelium, and an underlying fibrous connective tissue stroma. (C-F) IH findings (magnification X50, X100) of negative reactivity for Calretinin, Ki-67 as well as PCNA, further confirmed the diagnosis of a Dentigerous cyst and ruled out the possibility of a Unicystic Ameloblastoma. 

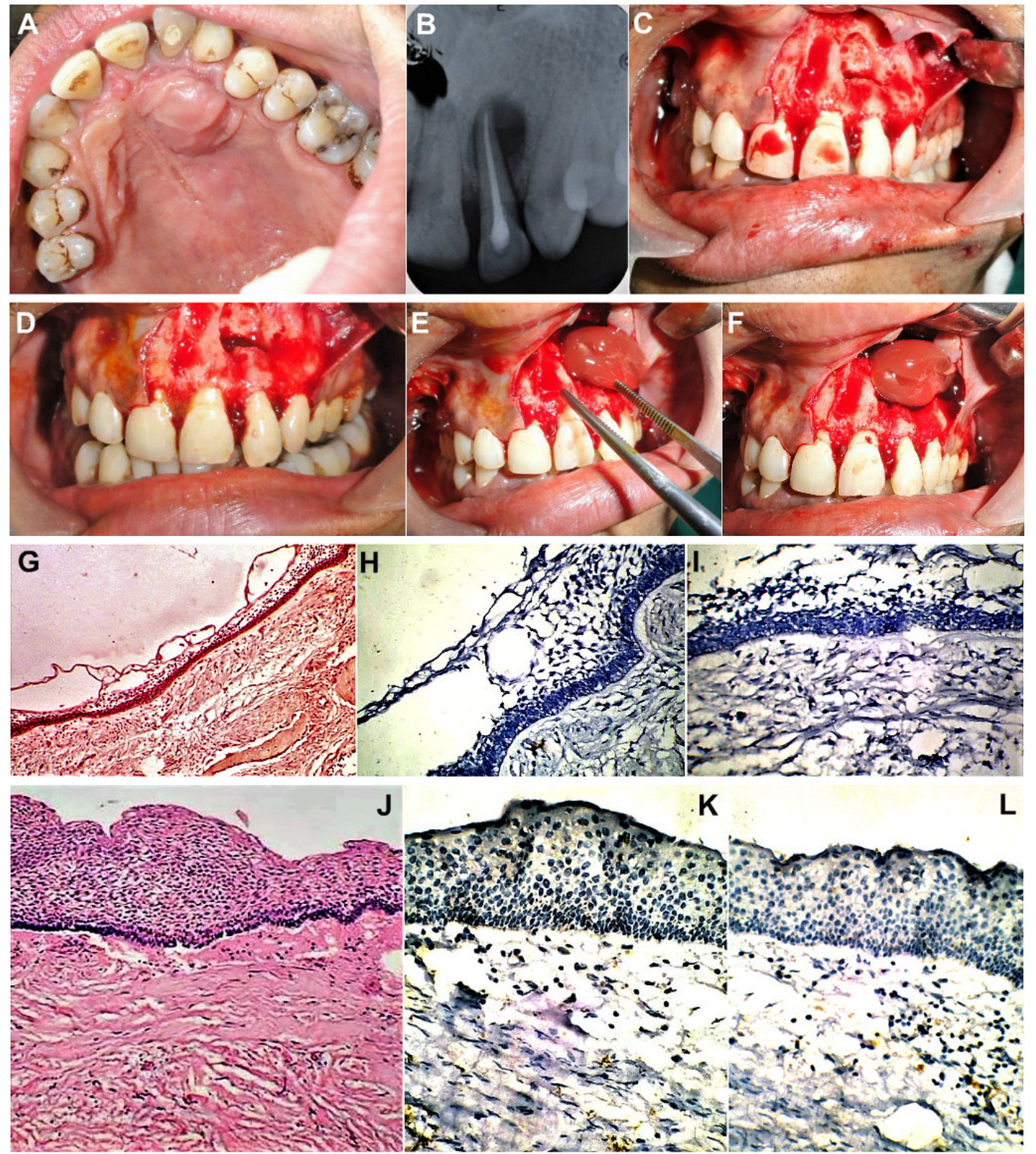

Figure 10: (Case 5) Large palatal cyst in a 15yr old patient seen in association with a non-vital upper left lateral incisor tooth. (B) Intraoral Periapical (IOPA) view radiograph showing a large unilocular radiolucency in the apical region of the involved tooth. Root canal treatment of the affected tooth completed. (C-F) Enucleation and curettage of the periapical pathology carried out under LA, followed by placement of fresh autologous PRF within the bony cavity. (G) H\&E Section (magnification X50). Epithelial lining adjacent to the cyst lumen, composed of varying thickness of stratified squamous epithelium, with underlying fibrous connective tissue. $(\mathrm{H}, \mathrm{I}) \mathrm{Negative} \mathrm{IH}$ expression of Calretinin (magnification X100). (J) H\&E Section (magnification X 1000). Areas of the cystic lesion showing thicker layers of stratified squamous epithelial lining. (K, L) IHC sections (magnification X1000) Corresponding sections of the epithelial lining, showing negative immunoreactivity for Ki-67 and PCNA, ruling out possibility of a more aggressive pathology.

Biotin Immunoperoxidase (ABI) staining technique was employed using Diaminobenzidine (DAB) as the chromogen for visualization of the Immunological complexes formed. Positivity was expressed as brown nuclear staining of the cells.

Steps in IHC Staining: Antigen retrieval of the Paraffin embedded tissue sections mounted on the slides, was carried out by the method of HIER (Heat Induced Epitope
Retrieval), using a microwave. Specimen were blocked to avoid unspecific cross reaction and background staining. Presence of endogenous enzymes, such as peroxidase, which are preserved in paraffin were blocked by incubating the sections with a mixture of $\mathrm{H}_{2} \mathrm{O}_{2}$ and methanol or incubating sections with $0.075 \% \mathrm{HCl}$ in ethanol at room temp for $15 \mathrm{~min}$ before immunostaining.

Thereafter, the slides with the tissue specimen were 

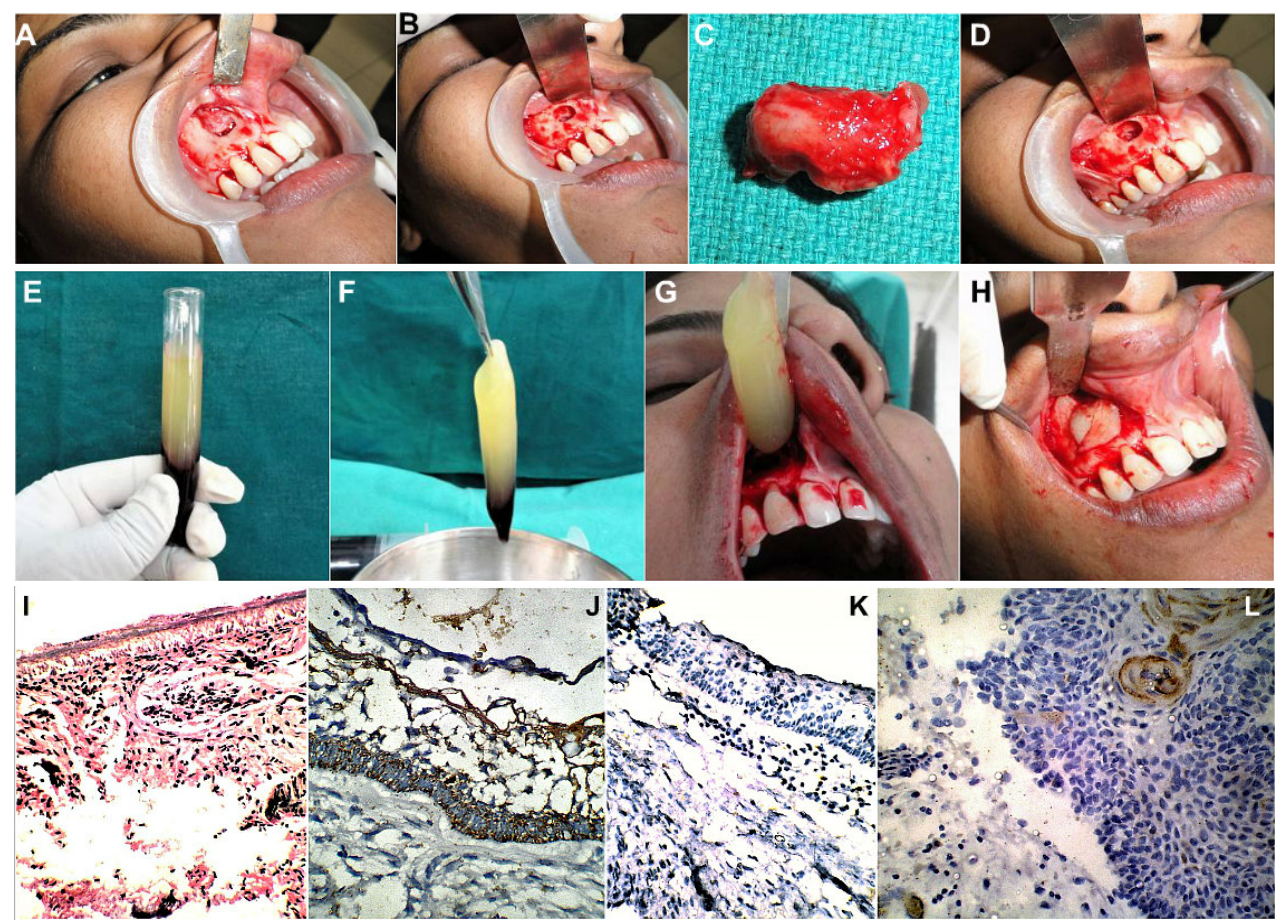

Figure 11: (Case 6): (A) Periapical cyst associated with a non-vital upper right lateral incisor, in a 16yr old female patient. (B-D) Root canal treatment of the involved tooth, followed by enucleation and curettage of the cystic lesion followed by apicoectomy was carried out under Local Anaesthesia. (E-H) Fresh autologous PRF was placed within the bony cavity to enhance subsequent bone fill and reossification of the lytic region. (I) H\&E sections (magnification X10) showed the cyst lumen lined with stratified squamous epithelium and surrounded by a fibrous connective tissue wall, both of which had a densely inflammatory cell infiltrate. $(\mathrm{J})$ A negative Calretinin expression(magnification X100), further helped to rule out the possibility of Unicystic Ameloblastoma. (H-I) Negative expression of Ki-67(magnification X50). (J, K) Negative expression of PCNA (magnification X100), indicative of a lesion of indolent growth potential.
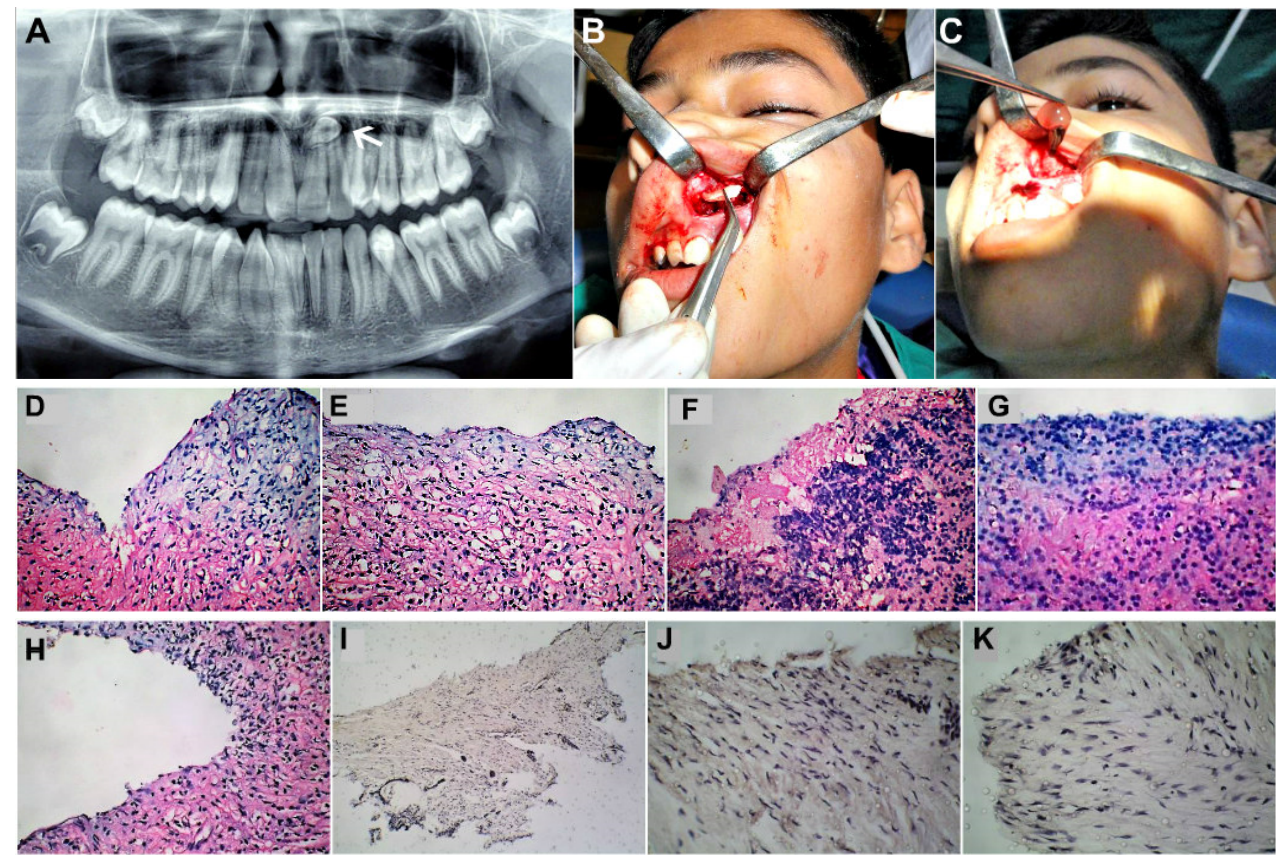

Figure 12: (Case 7) (A) OPG of an 8year old child showing a unilocular maxillary cystic lesion in association with an unerupted and displaced mesiodens between the roots of the upper central incisors. (B) Deroofing of the cystic lesion carried out via a labial sulcular approach, exposing the mesiodens, which was removed. (C) Enucleation and curettage of the lesion carried out. Fresh autologous PRF placed within the lytic space to encourage bone fill. (D-H) H\&E sections (magnification X50) showing histological features of a Dentigerous cyst. Cyst lumen was lined by stratified squamous epithelium and a dense fibrous connective tissue capsule containing chronic inflammatory cell infiltrate. (I) A negative Calretinin expression (magnification X100), further helped to rule out the possibility of Unicystic Ameloblastoma. (J) Negative expression of Ki-67(magnification X100). (K) Negative expression of PCNA (magnification X100). 
incubated overnight (12 hours) at $4^{0} \mathrm{C}$ with the Primary Monoclonal Antibody raised against the three Human Antigens of interest that were to be detected, namely, the Calretinin, Ki -67 Ag and Proliferating Cell Nuclear Antigen (PCNA). 25 to $50 \mu \mathrm{L}$ of diluted $\mathrm{Ab}$, a volume sufficient to completely cover the tissue was employed, so as to ensure that the tissue specimen would not dry out during incubation. Properly optimized working dilutions of the three Antibodies, in this case, 1:100 dilutions, were used.

Primary Mouse Monoclonal antibodies were used against Ki-67 (Clone MIB-1; 1:100 dilutions, Dako, Carpinteria, CA, USA) and PCNA (Clone PC10; dilution 1:100, Dako, Carpinteria, CA, USA). For Calretinin, optimally and precisely prediluted Rabbit Anti- Calretinin polyclonal primary antibody (Biogenex laboratories Inc., USA), was employed. The slide was then washed in phosphate buffered saline and incubated with the Secondary antibody, which is biotinylated, that is, conjugated with Biotin (Vitamin $\mathrm{H}$ / B7) and with the streptavidin/ peroxidase complex (LSAB p-labelled streptavidin-biotin, Dako), for $30 \mathrm{~min}$ each.

The Primary Ab was unlabeled, while the secondary Ab was the labelled Ab. An egg white protein called Avidin has a very strong affinity for Biotin, so an Avidin- Horseradish Phosphate (HRP) Complex was used as the reporter molecule which forms the ABI complex which can be visualized or developed using DAB as the chromogen which is turned brown by the HRP. Thus, the nuclei expressing Ki $67 \mathrm{Ag}$ and PCNA Ag turn brown.

As both Ki-67 and PCNA are Cell cycle associated antigens located within the nuclei, positivity was expressed as brown IHC Nuclear staining. Positivity for Calretinin, however, was expressed as both nuclear as well as cytoplasmic staining.

Calculation of LI / PI Values: The Ki-67 and PCNA Labelling Index (LI) or the Index of Positivity / Proliferation Index (PI) was calculated by counting the number of positive cells in five histopathological fields, dividing by the total number of cells in that field and multiplying by 100 .

\section{Results}

Confirmatory diagnosis for each destructive maxillary lesion was made by correlating the HPE and IHC analysis of the biopsy specimens (Table 1). Ten cases were diagnosed as Radicular / Periapical / Palatal cysts of inflammatory origin, associated with infected / non-vital teeth. These lesions demonstrated negativity for all three IHC markers. Eight cases were diagnosed as Dentigerous cysts associated with one or more impacted / unerupted teeth (Table 1). Two of these Dentigerous cysts showed histological as well as Immunohistochemical evidence of ameloblastomatous transformation, by demonstrating positivity for Calretinin as well as Ki-67 and PCNA, while the remaining six exhibited nil immunoreactivity. Two cases were diagnosed as Adenomatoid odontogenic tumors (AOT) / Adenoameloblastomas, demonstrating positivity for Calretinin but not for Ki-67 or PCNA. Two cases were diagnosed as Unicystic Ameloblastomas of the luminal variety and two cases as Odontogenic keratocysts (OKC) / Keratocystic odontogenic tumors (KCOT), all of them demonstrating positivity for all three IHC markers. One case was found to be a large Globulomaxillary cyst with negative IHC expression (Table 1).

Treatment plan was formulated on a case to case basis, based on the diagnosis established by correlating the histopathological presentation with the expression of the IHC Tumor Marker, namely, Calretinin. The treatment plan was also guided by the likely prognosis of each case, as indicated by the expression IHC Cell Proliferation Markers, namely Ki-67 and PCNA, by the constituent cells of the lesions.

The Inflammatory pathologies and Odontogenic cysts which exhibited negativity for all three IHC markers, were managed conservatively. Those cystic lesions undergoing ameloblastomatous transformation, the Unicystic Ameloblastomas, Keratocystic odontogenic tumors and Adenoameloblastomas, all of which exhibited positivity for the IHC markers, were managed more aggressively, in order to ensure their complete elimination, leaving behind no residual cells or satellite cysts, so as to prevent future

Table 1: Correlation of Histopathological (HPE) typing of 25 cases of large, destructive Unilocular Maxillary lesions in a Pediatric population, with their Immunohistochemical (IHC) expression of Calretinin (Tumor marker) and Labelling Indices of Ki-67 \& PCNA (Cell Proliferation Markers)

\begin{tabular}{|c|c|c|c|c|c|}
\hline S. No. & HPE diagnosis of the Maxillary pathology & Number of Cases & Calretinin Expression & KI-67 LI (Average LI) & PCNA LI (Average LI) \\
\hline 1. & Dentigerous Cyst & 06 & Negative & Negative (0) & Negative (0) \\
\hline 2. & $\begin{array}{l}\text { Dentigerous Cyst with evidence of Amelo- } \\
\text { blastic transformation }\end{array}$ & 02 & Positive & $1.1 ; 1.0(1.05)$ & $0.7 ; 0.8(0.75)$ \\
\hline 3. & Unicystic Ameloblastoma & 02 & Positive; Positive & $1.6 ; 0.9$ (1.25) & $1.6 ; 0.5$ (1.05) \\
\hline 4. & Globulomaxillary Cyst & 01 & Negative & Negative (0) & Negative (0) \\
\hline 5. & Radicular / Periapical Cyst & 10 & Negative & Negative (0) & Negative (0) \\
\hline 6. & $\begin{array}{l}\text { Adenoameloblastoma / Adenomatoid } \\
\text { Odontogenic Tumor (AOT) }\end{array}$ & 02 & $\begin{array}{l}\text { Positive; } \\
\text { Positive }\end{array}$ & $\begin{array}{l}\text { Negative (0) Nega- } \\
\text { tive (0) }\end{array}$ & $\begin{array}{c}\text { Negative (0) Negative } \\
(\mathbf{0})\end{array}$ \\
\hline 7. & Odontogenic Keratocyst & 02 & Positive & $0.9 ; 0.6(0.75)$ & $0.5 ; 0.5(0.5)$ \\
\hline
\end{tabular}


recurrence of the lesion. The Ki-67 and PCNA Proliferation Index (PI) / Labelling index (LI) values were relatively low, that is $<=1.5$, hence no case necessitated jaw resection / maxillectomy.

In the cases of unicystic ameloblastomas, the positivity for Calretinin, PCNA and KI-67 was exhibited mainly by the peripheral ameloblast-like cells lining the cystic cavity, followed by the areas of the epithelial islands within the cyst wall.

\section{Discussion}

Owing to the wide diversity in origin, etiopathogenesis and biological behaviour of different paediatric destructive maxillary pathologies, a precise and accurate diagnosis, followed by the most appropriate and effective management modality, that completely eliminates the lesion with least patient morbidity, is imperative for a successful esthetic and functional outcome. In this study IHC in conjunction with HPE, provided valuable information as to both, the diagnosis as well as the prognosis of these pathologies, contributing greatly to formulation of an ideal and efficacious treatment plan for each case.

In this Study, expression of Calretinin helped in distinguishing cystic lesions from odontogenic tumors, and also in identifying ameloblastomatous changes in Dentigerous cysts. This enabled implementation of the most appropriate surgical approach in each case.

Expression of 'Ki-67' and 'PCNA' was employed to assess the invasiveness, aggressiveness and recurrence potential of each of the paediatric maxillary pathologies in this Study. Negative expression of the IHC markers were suggestive of indolent and non-aggressive, slow growing pathologies, which were amenable to conservative treatment approaches, while positive IHC expression was suggestive of aggressive pathologies, meriting a more radial management approach to avoid possible recurrence.

In this Study of 25 cases of paediatric destructive, unilocular maxillary pathologies, at one end of the spectrum were those lesions which were found negative for all three IHC markers, thus ruling out a tumor, and indicative of a cystic lesion of low growth potential. These pathologies corroborated with the HPE findings of Periapical / Radicular cysts, Dentigerous cysts, Primordial and Globulomaxillary cysts. On the basis of the prognosis indicated by the HPE and IHC assessment, these pathologies were treated effectively using a conservative surgical approach of enucleation and curettage, accompanied by extirpation of the maxillary antral lining in those cases where the cyst lining was found contiguous with the lesion. The conservative approach ensured a successful elimination of the pathology, while at the same time producing the least functional morbidity and esthetic deformity.
At the other end of the spectrum of this Study, were those paediatric maxillary pathologies, which were found positive for all three IHC markers, and corroborated with the histological typing of Unicystic ameloblastomas or Dentigerous cysts undergoing ameloblastomatous transformation or OKCs. These lesions exhibited positivity for the Tumor marker, Calretinin, as well as the Cell proliferation markers, namely, Ki-67 and PCNA, albeit exhibiting low Labelling Index values $(<1.5)$. These lesions were managed more aggressively, by enucleation, curettage, extirpation of the antral lining, peripheral ostectomy of the bony walls of the cavitation and chemical cauterisation of the antral walls with Carnoy's solution. The relatively aggressive treatment approach enabled complete elimination of the lesion, and also ensured minimising all possible chances of recurrence of the pathology, which would otherwise have necessitated repeat surgeries and possible future cosmetic deformity and functional debility.

All the cases of Periapical / Radicular and Palatal cysts (IHC Labelling Index Value $=0$ ) were managed conservatively, by Root Canal Therapy of the involved tooth, followed by enucleation and curettage of the cystic lesion (Cases 5, 6 \& 7; Figures 10, 11 \& 12). This was followed by placement of fresh autologous Platelet Rich Fibrin within the bony cavity to enhance soft tissue healing as well as hasten subsequent reossification and bone fill.

Enucleation and curettage was performed for the Globulomaxillary cyst; Enucleation and curettage, accompanied by extirpation of the maxillary antral lining, and placement of PRF was carried out for the cases of Dentigerous cysts (Case 4; Figures 7, 8 \& 9). Enucleation and curettage, antral lining extirpation, antral lavage, followed by chemical cauterization of the antral walls with Carnoy's solution was employed for the cases showing positivity for the IHC markers, which included Odontogenic tumors such as Adenoameloblastoma, Unicystic Ameloblastomas (Case 2; Figures 3 \& 4), Odontogenic Keratocyst (OKC) (Case 3; Figures 5 \& 6) and for those Dentigerous cysts showing evidence of ameloblastomatous transformation (Case 1; Figures 1 \& 2).

As, there was no case of malignant tumors or benign, aggressive pathologies with medium (LI $>1.5$ ) or high $\mathrm{IH}$ Labelling Index ( $\mathrm{LI}>3$ ) values, encountered in this study, no case was indicated for a more aggressive management protocol such as partial or complete maxillectomy.

There was no incidence of recurrence of the pathology in any of the 25 cases, during the entire follow up period, which ranged from one to two years. Excellent functional and esthetic outcomes were achieved in all the cases, with nil incidence of morbidity or functional debility. There were no neurological deficits observed in any case. Minor 
post-operative complications such as pain, swelling and edema resolved within three to five days following surgery. A superior bone fill and reossification of the lytic areas, was observed in those cases where fresh, autologous PRF had been placed intraoperatively, within the operative site prior to closure.

In this study, the IHC analysis of the biopsy specimen was corroborated with that of the final excised / enucleated specimen in all the cases, with no discrepancy in results noted in any case. Taking care to obtain a representative sample of the pathology at the time of biopsy, is essential for an accurate diagnosis to be made, and for the most ideal and effective treatment procedure to be implemented. A possible risk during biopsy of such destructive maxillary pathologies, could be to miss out the section of a Dentigerous cyst that might be undergoing ameloblastomatous transformation, and hence underreporting of the specimen with the subsequent treatment rendered, being more conservative than desired. Hence, a thorough histopathological and IHC examination of the entire pathology following excision is important for confirmation of the diagnosis.

A possible limitation of this Study could be the limited number of Cases observed and treated. Another drawback is that Immunohistochemistry is not always infallible as it is an intricate and technique sensitive lab procedure, where mistakes can occur during tissue processing and errors in antigen retrieval, yielding false positive or negative results. Hence, IHC must be viewed as a valuable adjunct to, rather than a substitute for Light microscopy.

\section{Conclusion}

Immunohistochemical analysis served as a valuable adjunct to histopathogical examination, in the management of 25 cases of destructive maxillary pathologies in the paediatric population. Calretinin served as a reliable IHC diagnostic marker, which helped in reaching a precise confirmatory diagnosis. Ki-67 and PCNA served as useful predictive and prognostic indicators, by providing valuable information about the nature of the lesions, in terms of their biological behavior, aggressiveness and tendency for recurrence. Armed with this information, the surgeon was able to make an informed and sound clinical decision, and employ the most efficacious management protocol for each case. This helped in achieving the best functional and esthetic outcomes in all the 25 cases, with the least possible morbidity, deformity or debility, and nil incidence of recurrence in any of the affected children.

\section{Compliance With Ethical Standards}

\section{Disclosure of potential Conflicts of Interest}

The author of this article has not received any research grant, remuneration, or speaker honorarium from any company or committee whatsoever, and neither owns any stock in any company. The author declares that he / she does not have any conflict of interest.

\section{Research involving human participants and /or animals}

All procedures performed on the patients (human participants) involved were in accordance with the ethical standards of the institution and/or national research committee, as well as with the 1964 Helsinki declaration and its later amendments and comparable ethical standards.

Ethical approval: This article does not contain any new studies with human participants or animals performed by the author.

Informed Consent: Informed consent was obtained from all the individual participants in this study, including parents of the minors.

\section{Funding}

This study was not funded by any organization / society.

\section{References}

1. R Scariot, RV da Silva, W da Silva Felix Jr., DJ da Costa, NLB Rebellato. "Conservative treatment of ameloblastoma in child: a case report," Stomatologija, 2012; 14 (1): 33-6.

2. Gulbranson SH, Wolfrey JD, Raines JM, McNally BP. Squamous cell carcinoma arising in a dentigerous cyst in a 16-month-old girl. Otolaryngol Head Neck Surg 2002; 127 (5) :463-4. DOI:10.1067/ mhn.2002.129039

3. Dunsche A, Babendererde O, Luttges J, et al. Dentigerous cyst versus Unicystic Ameloblastoma-differential diagnosis in routine histology. J Oral Pathol Med. 2003; 32 (8):486-91. PMID: 12901731. DOI: $10.1034 /$ j.1600-0714.2003.00118.x

4. Sandra F, Mitsuyasu T, Nakamura N, Shiratsuchi Y, Ohishi M. Immunohistochemical evaluation of PCNA and Ki-67 in Ameloblastoma. Oral Oncol 2001; 37 (2):193-8. PMID: 11167148. DOI: $10.1016 / \mathrm{s} 1368-8375(00) 00079-8$

5. Coleman H, Altini M, Ali H, Doglioni C, Favia G, Maiorano E. Use of calretinin in the differential diagnosis of unicystic ameloblastomas. Histopathology 2001; 38 (4); 312-7. PMID: 11318896. DOI: $10.1046 / j .1365-2559.2001 .01100 . x$

6. Meer S, Galpin JS, Altini M, Coleman H, Ali H. Proliferating cell nuclear antigen and Ki-67 immunoreactivity in ameloblastomas. Oral Surg Oral Med Oral Pathol Oral Radiol Endod 2003;95 (2):213-21. PMID: 12582363. DOI: $10.1067 /$ moe.2003.62

7. Kimi K, Kumamoto H, Ooya K, Motegi K. Immunohistochemical analysis of cell cycle and apoptosis-related factors in lining epithelium of odontogenic keratocysts. J Oral Pathol Med 2001: 30 (7): 434-42. PMID: 11488422. DOI: 10.1034/j.1600-0714.2001.300709.x

8. Alaedinni M, Etemad-Moghadam S, Baghaii F. Calretinin in Odontogenic tumors. Histopathology 2008; 52 (3): 299-304. PMID: 18269580. DOI: $10.1111 / \mathrm{j} .1365-2559.2007 .02948 . x$

9. De Villiers P, Liu H, Suggs C, et al. Calretinin expression in the differential diagnosis of human ameloblastoma and keratocystic 
odontogenic tumor. Am J Surg Pathol. 2008:32 (2):256-60. PMID: 18223328. DOI: 10.1097/PAS.0b013e3181452176

10. Chapelle KA, Stoelinga PJ, Wilde PC, Brouns JJ, Voorsmit RA. Rational approach to diagnosis and treatment of ameloblastomas and odontogenic keratocysts. Br J Oral Maxillofac Surg 2004; 42
(5):381-90. PMID: 15336762. DOI: 10.1016/j.bjoms.2004.04.005

11. Rosenstein T, Pogrel MA, Smith RA, Regezi JA. Cystic ameloblastomaBehaviour and treatment of 21 cases. J Oral Maxillofac Surg. 2001; 59 (11):1311-6. PMID: 11688034. DOI: 10.1053/joms.2001.27522

12. H Kumamoto, K Kimi, K Ooya. Detection of cell cycle-related factors in ameloblastomas. J Oral Pathol Med 2001; 30 (5):309-15. https://doi. org/10.1034/j.1600-0714.2001.300509.x 